\title{
Emergence of de novo cutaneous vasculitis post coronavirus disease (COVID-19) vaccination
}

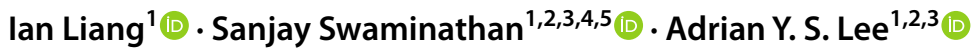

Received: 10 September 2021 / Revised: 20 September 2021 / Accepted: 29 September 2021 / Published online: 2 October 2021

(c) Crown 2021

\section{Presentation}

A 62-year-old Asian female presented to the Emergency Department with a bilateral lower limb non-blanching petechial rash 7 days after the first dose of the ChAdOx $1 \mathrm{nCoV}$ 19 vaccine (Astra-Zeneca) COVID-19 vaccination (Fig. 1A). Her symptoms were associated with a generalised headache, myalgia, and symmetrical large joint arthralgias. She was afebrile, had no appreciable synovitis, and the rest of the physical examination was unremarkable.

Her haematological and biochemical pathology profiles were unremarkable including preserved renal function. Urinalysis revealed trace leukocytes, haemolysed blood but with no dysmorphic red blood cells on microscopy. A spot urine protein:creatinine ratio was within normal limits. Her CRP was $31 \mathrm{mg} / \mathrm{L}(<5)$. An autoimmune workup revealed a lowtitre antinuclear antibody (1:80 speckled), no anti-extractable

Adrian Y. S. Lee

adrian.lee1@health.nsw.gov.au

Ian Liang

ian.liang@health.nsw.gov.au

Sanjay Swaminathan

sanjay.swaminathan@health.nsw.gov.au

1 Department of Clinical Immunology, Westmead Hospital, Westmead, NSW, Australia

2 Department of Immunopathology, NSW Health Pathology \& ICPMR, Westmead Hospital, Westmead, NSW, Australia

3 Sydney Medical School, Westmead Hospital, Westmead, NSW, Australia

4 Centre for Allergy and Immunology Research, The Westmead Institute for Medical Research, Westmead, NSW, Australia

5 School of Medicine, Western Sydney University, Westmead, NSW, Australia nuclear antigen antibodies, no anti-neutrophil cytoplasmic antibodies (ANCA), and raised rheumatoid factor (169 IU/ $\mathrm{mL}[<20])$ with depressed C4 complement $(<0.07 \mathrm{~g} / \mathrm{L})$. Cryoglobulins and anti-cyclic citrullinated peptide antibodies were not detected. An infectious screen including hepatitis and syphilis serologies was unremarkable.

A CT pulmonary angiogram did not reveal pulmonary emboli, and an MRI brain venogram did not demonstrate a cerebral sinus venous thrombosis. Skin punch biopsies revealed perivascular acute inflammation (Fig. 1B) with C3 and fibrinogen deposition in the superficial dermal vessels on immunofluorescence microscopy (Fig. 1C, D), consistent with a leukocytoclastic vasculitis. The patient was commenced on a rapid tapering course of oral prednisolone to good resolution and improvement in her symptoms, rash, and pathology tests. No underlying systemic autoimmune disease was established.

\section{Discussion}

There have been numerous reports in literature where vaccines such as the influenza and the Bacille Calmette-Guerin vaccines have resulted in vasculitis as an adverse reaction [1]. Reports also exist where the COVID-19 vaccination has caused a flare of pre-existing leukocytoclastic vasculitis [2] and the development of $\operatorname{IgA}$ vasculitis in a patient with prior COVID-19 illness [3]. The exact aetiology of postvaccination vasculitis is unknown, but risk factors include genetic, immunological, hormonal, and environmental factors [4]. Whilst the vaccine was certainly contributory to the development of vasculitis in this patient, it is possible that it was triggered in an already immunologically predisposed individual [5]. It is therefore important that clinicians are aware that vasculitis can be a possible adverse reaction to the ChAdOx $1 \mathrm{nCoV}-19$ vaccine, both de novo and pre-existing. 
Fig. 1 Leukocytoclastic vasculitis following coronation. Non-blanching bilateral lower limb vasculitic rash (A). On microscopy, perivascular inflammation was noted (arrow) (B) with deposition of $\mathrm{C} 3$ complement (C) and fibrinogen (D) in the superficial dermal vessels. Micrographs are taken at $100 \times$ magnification virus (COVID-19) vaccina-
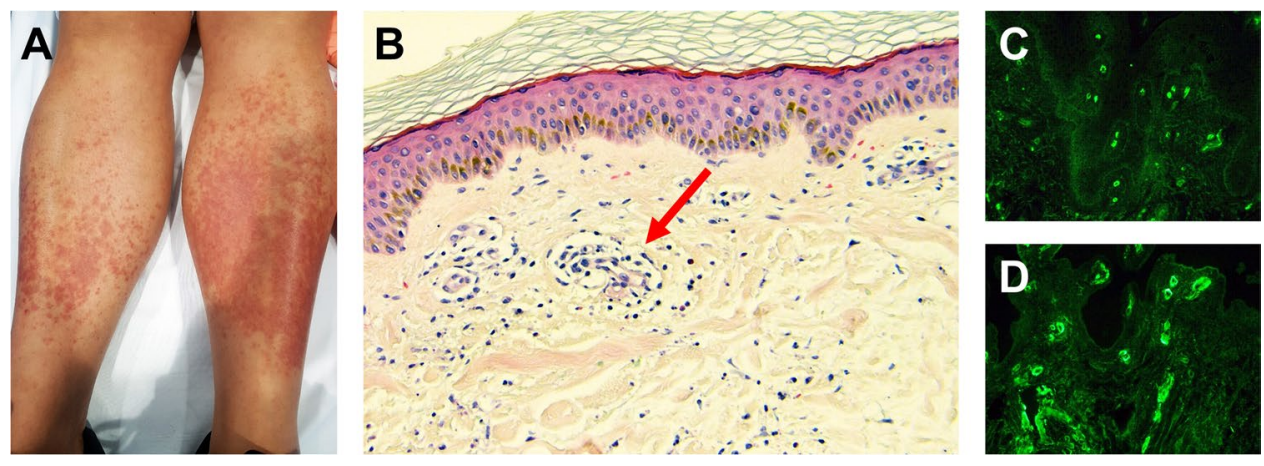

following immunization — systematic literature review. Vaccine 34(51):6641-6651. https://doi.org/10.1016/j.vaccine.2015.09.026

or contribution IL contributed to the manuscript draft, concept design, data acquisition and analysis, and clinical care of the patient. SS contributed to the manuscript draft, concept design, supervision, and clinical care of the patient. AL contributed to the manuscript draft, concept design data acquisition and analysis, and clinical care of the patient. All authors have contributed to revising the manuscript critically for intellectual content.

\section{Declarations}

Ethics approval and consent to participate Written informed consent was obtained from the patient for publication of this case report and any accompanying images.

Disclosures None.

\section{References}

1. Bonetto C, Trotta F, Felicetti P, Alarcón GS, Santuccio C, Bachtiar NS et al (2016) Vasculitis as an adverse event
2. Cohen SR, Prussick L, Kahn JS, Gao DX, Radfar A, Rosmarin D (2021) Leukocytoclastic vasculitis flare following the COVID-19 vaccine. Int J Dermatol 60(8):1032-1033. https://doi.org/10.1111/ ijd. 15623

3. Bostan E, Gulseren D, Gokoz O (2021) New-onset leukocytoclastic vasculitis after COVID-19 vaccine. Int J Dermatol. https://doi. org/10.1111/ijd.15777

4. Molina V, Shoenfeld Y (2005) Infection, vaccines and other environmental triggers of autoimmunity. Autoimmunity 38(3):235245. https://doi.org/10.1080/08916930500050277

5. Akinosoglou K, Tzivaki I, Marangos M (2021) Covid-19 vaccine and autoimmunity: awakening the sleeping dragon. Clin Immunol. 226:108721. https://doi.org/10.1016/j.clim.2021.108721

Publisher's note Springer Nature remains neutral with regard to jurisdictional claims in published maps and institutional affiliations. 